\title{
RECHERCHES SUR LE MÉCANISME DE LA TRANSMISSION DES FILAIRES PAR LES CULICIDÉS
}

\author{
Par Henri GALliaRD
}

$\mathrm{Au}$ cours des recherches que nous avons eu l'occasion d'effectuer sur l'évolution de Filaria bancrofti, F. malayi, Dirofilaria immitis, avec différentes espèces de culicidés ou d'anophèles, nous avons pu étudier le mode d'émergence par la trompe des larves infestantes ainsi que les conditions qui favorisent ce phénomène.

On sait que la migration des larves de Wuchereria bancrofti des muscles thoraciques dans le labium a été découverte en rgoo par Low, James, Grassi et Noe. Annett, Dutton et Elliott (rgor) pensèrent, sans le démontrer, que la larve pouvait rompre la membrane qui se trouve à l'exırémité du labium et relie les labelles, et qu'ils appelèrent membrane de Dutton.

Lebredo, en ז905, montra que le passage s'effectuait en effet par rupture de cette membrane. Ces observations furent confirmées par Fülleborn ( 1905 ) et Bahr ( I 9 I 2 ).

Cependant, Mochizuki en rg ro montra que le processus n'était pas celui qui était généralement admis jusque-là. Il observa en effet que les larves de la filaire émergeaient de la trompe en perçant l'extrémité des labelles. Mais ses observations publiées en japonais passèrent inaperçues.

C'est en r 926 que Yamada et Komori, étudiant la réceptivité de différentes espèces de Culex ou d'anophèles, résolurent expérimentalement le problème. Ces auteurs montrèrent, par des coupes en série, que la lumière du labium communique avec celle des labelles par un orifice suffisant pour le passage d'une seule larve à la fois. Il existe d'autre part, à l'extrémité des labelles, une zone de moindre résistance. Lorsque l'on exerce une pression sur les labelles avec une aiguille fine ou une lamelle, on voit cet organe se diviser à son apex en trois valves.

Yamada et Komori ont montré qu'on pouvait facilement réaliser ce processus de rupture, en coupant le labium près de la tête et en Annales de Parasitologie, T. XVIII, Nos 4-5-6. - 1941, p. 209-214. 
introduisant un crin très fin qui passe aisément à l'intérieur de l'articulation réunissant le labelle et le labium et détermine la séparation des pièces constituant le labelle.

Dans leurs expériences, ils coupaient la tête du Culicidé. En exerçant sur la trompe une pression très légère avec le côté d'une aiguille ou en déposant simplement une lamelle sur la trompe (Bancroft), on détermine une excitation de la larve et les auteurs ont constaté que les larves sortaient toujours par l'extrémité des labelles.

Sundar Rao et Iyengar ont confirmé les recherches de Yamada et Komori en démontrant que la sortie de la larve se faisait exactement de la même façon par ce même processus dans les conditions naturelles sans qu'on exerce aucune pression, ni qu'on fasse intervenir des modifications de la température. En étudiant le processus de pénétration des larves infestantes à travers la peau, ces auteurs firent gorger sur un volontaire des Culex déjà expérimentalement infestés. Ln examinant la trompe d'un des spécimens, ils virent une larve qui effectuait sa sortie par l'extrémité des labelles.

Nous avons fait, dans un très grand nombre de cas, les mêmes constatations. La figure 5, pl. XIII, montre une larve de Wachereria bancrofti émergeant d'un labelle de Culex fatigans.

La figure I montre une larve de Dirofilaria immitis sortant, chez Aedes albopictus, du labelle gauche. L'extrémité du labelle droit est déjà rompue, une larve ayant déjà eu le temps de sortir avant la fixation. Une troisième larve est encore dans le labium, prête à passer dans le labelle.

Parfois cependant, la larve n'arrive pas à rompre l'extrémité des labelles. II arrive qu'elle décrive une boucle à leur intérieur, revienne dans le labium et ressorte par l'autre labelle comme on le voit sur la figure 5 .

Sur les figures 3 et 4 , on voit que parfois les larves peuvent décrire une boucle dans chacun des labelles et revenir ensuite, après avoir échoué dans leurs tentatives de rupture, dans le labium. Dans ce cas, une troisième larve essaye de passer également dans le labelle gauche.

Ajoutons que ces photographies concernent des Culicidés examinés entiers et sans compression, fixés dans l'alcool dès que les larves commençaient à sortir. Il est manifeste que lorsque l'on sectionne l'insecte au niveau du cou, les larves attirées par l'humidité de l'air quittent le labium pour sortir immédiatement de la tète. La sortie se fait encore plus rapidement si la tète et la trompe sont placées dans une goutte d'eau. Quand on sectionne la trompe, la sortie des larves est immédiate (fig. 4 et 6). Yamada et Komori ont pourtant observé 
que les larves ne s'échappaient pas du labium avant 2 à 5 heures, mème en irriguant l'extrémité. C'est ce qui se passe quand l'insecte est intact, à moins qu'on n'élève le degré de température ou d'humidité.

Nous avons observé aussi que le chloroforme peut déterminer, tout au moins pendant les premières minutes, une excitation particulière des larves dans la trompe à tel point qu'elles sont déjà en voie de sortie au moment où l'on place l'insecte anesthésié sous le binoculaire (fig. r). L'agitation et les chocs sur les parois d'un tube sont insuffisants pour déterminer le mème phénomène.

Il est donc nécessaire que la larve reçoive une excitation d'ordre quelconque pour qu'elle effectue les mouvements de rupture. Cette excitation est probablement et surtout d'ordre mécanique. Car il ne nous semble pas, d'après des expériences répétées, que le simple contact de la peau humaine ou animale puisse avoir une influence quelconque.

Dans les conditions naturelles, il est certain que c'est la flexion de la lèvre inférieure au moment de l'introduction des pièces buccales dans la peau qui doit agir au premier chef. Rao et Iyengar ont observé en effet la sortie immédiate des larves chez des spécimens de Culex fatigans, déjà infestés par Wuchereria bancrofti et gorgés incomplètement sur l'homme.

La proportion des insectes infestés doit donc diminuer au fur et à mesure que le temps passe et finir par devenir nulle quand on a dépassé le temps normal de l'évolution des larves pour une température donnée. En maintenant, en efiet, les Culicidés à la température optima pour le développement des filaires, nous n'avons jamais pu réussir à les empêcher de se débarrasser de leurs larves. Ainsi à $30^{\circ}-35^{\circ}$, les larves infestantes sont dans la trompe au bout de 9 à Io jours; à $26^{\circ}-3 \mathrm{I}^{\circ}$, au bout de 17 jours. Nous n'avons retrouvé cependant de larves que pendant 7 jours après ces dates.

Mais il semble que ce ne soit pas uniquement l'acte de se gorger sur l'animal ou l'homme qui détermine cette élimination. Nous pensons, autant que l'on peut en juger par une seule expérience, que les femelles perdent aussi bien et aussi rapidement leur infestation quand elles sont nourries d'eau sucrée que lorsqu'elles se gorgent sur cobaye. Yamada et Komori, avec des Ciulex pipiens infestés et conservés dans des tubes à essai avec du coton imbibé deau sucrée, avaient montré d'ailleurs que le nombre des moustiques infestés diminuait de jour en jour et que le vingt et unième jour, toutes les femelles survivantes étaient indemnes de parasites, et leurs labelles étaient rompus.

$\mathrm{Hu}$, en 1935 , a signalé qu'il avait trouvé des Culex pipiens infestés 
avec $W$. bancrofti de 40 à $9^{3}$ jours après le repas infestant, et $6_{7}$ jours après pour $D$. immitis. Il est vrai que ces expériences ont été faites dans des conditions spéciales, la température ayant considérablement baissé après les $\mathrm{i} 5$ premiers jours et les moustiques ayant été nourris de suc de raisin. Il est évident que, dans ces conditions, à basse température, l'activité des larves matures est considérablement diminuée et il est donc tout à fait naturel qu'elles restent indéfiniment dans l'organisme de l'insecte tant qu'il est en vie, sans chercher à s'en échapper.

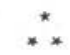

Aedes aegypti et $A$. albopictus, qui sont de petite taille, présentent normalement dans la trompe quatre ou cinq larves de $D$. immitis. Nous avons trouvé une fois 13 larves dans la trompe d'A. aegypti. Dans certaines circonstances, il semble donc possible que le labium, dilaté par plusieurs larves, puisse se rompre et que la sortie des larves puisse s'effectuer en un point quelconque. La figure 4 montre deux larves sortant de la face ventrale du labium, au niveau de son tiers antérieur, d'un Aedes albopictus, trouvé mort dans la cage d'expérience. Il suffit d'ailleurs de voir combien le vol de ces moustiques, de taille relativement petite, est lourd, sautillant et maladroit quand ils sont ainsi infestés, pour comprendre qu'ils puissent traumatiser leur trompe contre les parois de verre, ou bien en faisant effort pour se gorger.

Il semble bien prouvé cependant que l'infestation ne puisse nuire à la vitalité de l'insecte, bien que Bahr aux Fidji (19r2) ait constaté que la survie des spécimens d'A. variegatus infestés était très courte comparativement à celle des témoins. Par contre, Hick en Sierra Leone $\left(\mathrm{r} 9^{32}\right)$ n’a constaté aucune différence en expérimentant avec des anophèles.

Nous avons fait des observations identiques avec $C$. fatigans pour $W$. bancrofti, et avec A. aegypti, A. albopictus pour D. immitis, confirmant ainsi les recherches entreprises à Shangaï par Hu et Yen ( $\left.19^{33}\right), \mathrm{Hu}\left(\mathrm{r} 9^{35}\right)$ avec Culex pipiens et $W$. bancrofti.

\section{RÉsumé}

Dans cette note, nous indiquons quelques particularités du mécanisme de la migration des larves de Wuchereria bancrofti et Dirofilaria immitis, hors du labium de Culex fatigans, d'Aedes aegypti ou 
d'A. albopictus ainsi que les causes paraissant déterminer cette miğration.

L'action mécanique de la flexion de la trompe au moment du repas de sang semble devoir en être la cause principale. Cependant, l'élimination des larves infestantes semble parfois se produire aussi rapidement chez les moustiques nourris d'eau sucrée. Ces larves ne persistent en effet que quelques jours après la durée normale de l'évolution pour une température donnée, à condition qu'elle soit maintenue après ce délai. Si elle s'abaisse, les larves mûres persistent indéfiniment. L'infestation massive de la trompe ( 13 larves de $D$. immitis chez un spécimen d'Aedes aegypti) peut en déterminer parfois la rupture, mais en général la longévité des moustiques infectés est identique à celle des témoins.

\section{Bibliographie}

Anmett (H. E.), Dutron (J. E.) et Elliot (J. H.). - Report of the malaria expedition to Nigeria, part. II, Filariasis. Liverpool School of Trop. Med., Memoir 4, r ror.

Bancroft (Th. L.). - Preliminary notes on the intermediary host of Filaria immitis Leidy. Journ. Trop. Med., IV, rgor, pp. 347-349.

Barr (P. H.). - Filariasis and Elephantiasis in Fiji. Journ. of London School of Trop. Med., Suppl. I, I912.

Feng (L. C.). - Anopheles hyrcanus var. sinensis Wied,, transmitter of Wuchereria (Filaria) bancrofti in Woosung district, Shanghaï. Amer. Jl. Hyg., XIV, I93I, pp. 502-5 I4.

Fülleborn (F.). - Ueber Versuche an Hundefilarien und deren Uebertragung durch Mücken. Archiv. f. Schiffs- $u$. Tropenhyg., XII, r9o8, Beiheft 8, pp. 3r3$35 \mathrm{r}, 4 \mathrm{pl}$.

Hıcкs (E. P.). - The transmission of Wuchereria bancrofti in Sierra Leone. Ann. of Trop. Med. and Parasit., XXVI, 1932, pp. 407-422.

Hu (S. M. K.). - Studies on host-parasite relationships of Dirofilaria immitis Leidy and its Culicine intermediate hosts. Amer. Jl. Hyg., XIV, I93r, pp. 6r4-629.

Hu (S. M. K.) et YEN (C. H.). - Studies on the susceptibility of Culex pipiens, var. pallens Coq. with Wuchereria bancrofti Cobb. in Woosung district. Chinese Med. Jl., XLVII, I933, pp. 1367-1372.

- Studies on the comparative susceptibility of Culex pipiens, var. pallens Coq. and Culex fatigans Wied. to experimental infection with Wuchereria bancrofti Cobb. Trans. $9^{\text {th }}$ Congress Far East Ass. Trop. Med., I, 1934, pp. 483-490.

Grassi (B.) et Noe (G.). - The propagation of the filariae of the blood exclusively by means of the puncture of peculiar mosquitoes. Brit. Med. Journ., II, 1900, pp. 1306-1307.

JAMES (S. P.). - On the metamorphosis of the Filaria sanguinis hominis in mosquitoes, etc. Brit. Med. Journ., II, 1900, pp. 533-537. 
Lebredo Mario (G.). - Metamorphosis of Filaria in the body of the mosquito (Culex pipiens). Journ. Infect. Dis., Suppl. I, rgo5 pp. 332-352.

Low (G. G.). - A recent observation on Filaria nocturna in Culex : probable mode of infection of man. Brit. Med. Journ., I, r9oo, pp. 1456-1 457 .

Mocmizukı (D.). - On the larvae of Filaria bancrofti (translation). Fukuoka Ikadaigaku Zasshi, III, I900, pp. 435-486.

Rao (S. S.) et Iyengar (M. O. T.). - The escape of the Filaria larva from the proboscis of Culex fatigans. Indian Journ. Med. Research, XIX, 1932, pp. $94 \mathrm{r}-943$.

Yamada (S. I.) et Kamewe Komori. - From what point of the proboscis of the mosquito do developed larvae of Filaria bancrofti escape? Scientific Reports from the Government institute for Infect. Diseases, V, 1926, pp. 647-65x.

$\mathrm{Y}_{\mathrm{BN}}$ (C. H.). - Note on the periodicity of Wuchereria bancrofti Cobb, in Shanghaï region. Lingnan Sc. Jl., XIII, I934, pp. 607-613.

\section{Laboratoire de Parasitologie}

de l'Ecole de Médecine de l'Indochine (Hanoï)

(Directeur : Prof. H.-G. Galliard).

\section{EXPLICATION DE LA PLANCHE XIII.}

FIG. 1. - Larve de Dirofilaria immitis chez Aedes albopictus.

Fı. 2. - Larves de Dirofilaria immitis bloquées dans les labelles de Aedes albopictus.

Fig. 3. - Deux larves de Dirofilaria immitis trouvées émergeant à moitié du labium spontanément rompu d'Aedes albopictus.

FIg. 4. - Deux larves de Dirofilaria immitis sortant de la face ventrale du labium, chez un Aedes albopictus trouvé mort.

Fig. 5. - Larve de Wuchereria bancrofti émergeant du labelle de C. fatigans.

Fig. 6. - Larves de Dirofilaria immitis sortant de la trompe sectionnée ou rompue d'Aedes albopictus. 
ANNALES DE PARASITOLOGIE

T. XVIII, Nos 4-5-6, 1941
Planche XIII

(Mémoire Galliard)
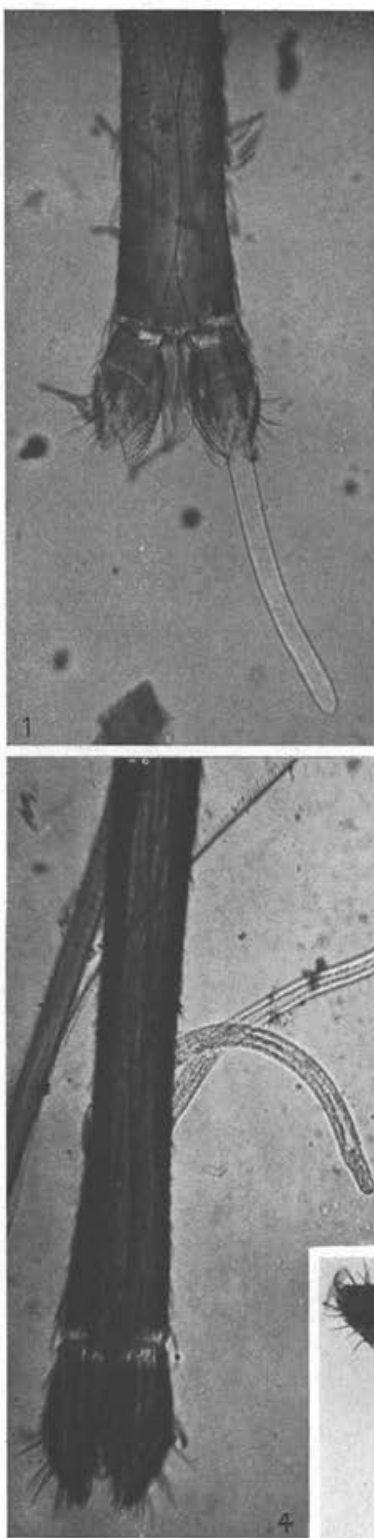
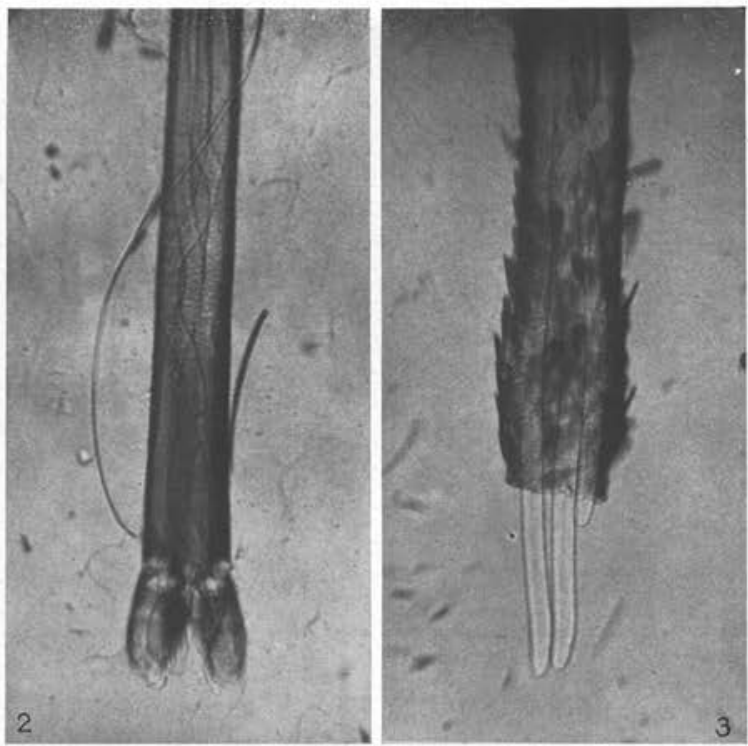

3

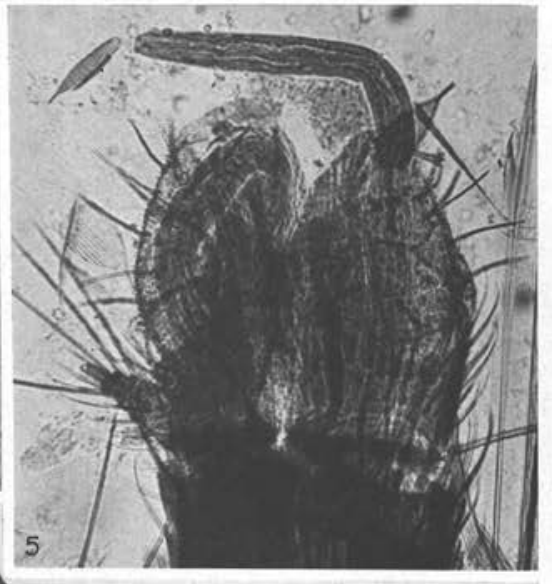

6

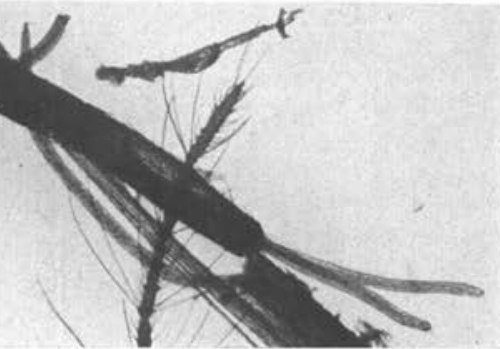

Masson et Cie, Éditeurs 